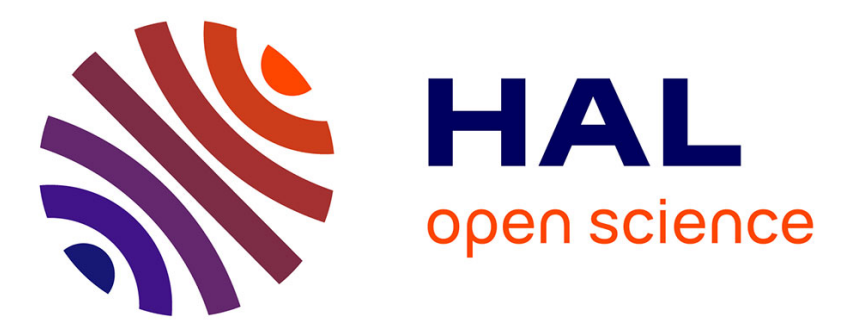

\title{
Comparison between centrifugal partition chromatography and preparative liquid chromatography as first dimensions in off-line two-dimensional separation: Application to the isolation of multi-targeted compounds from Edelweiss plant
}

Léa Marlot, Magali Batteau, Karine Faure

\section{To cite this version:}

Léa Marlot, Magali Batteau, Karine Faure. Comparison between centrifugal partition chromatography and preparative liquid chromatography as first dimensions in off-line two-dimensional separation: Application to the isolation of multi-targeted compounds from Edelweiss plant. Electrophoresis, 2018, 39 (15), pp.2011-2019. 10.1002/elps.201800032 . hal-01809502

\author{
HAL Id: hal-01809502 \\ https://hal.science/hal-01809502
}

Submitted on 23 Jul 2020

HAL is a multi-disciplinary open access archive for the deposit and dissemination of scientific research documents, whether they are published or not. The documents may come from teaching and research institutions in France or abroad, or from public or private research centers.
L'archive ouverte pluridisciplinaire HAL, est destinée au dépôt et à la diffusion de documents scientifiques de niveau recherche, publiés ou non, émanant des établissements d'enseignement et de recherche français ou étrangers, des laboratoires publics ou privés. 
1 Comparison between centrifugal partition chromatography and preparative liquid

2 chromatography as first dimensions in off-line two-dimensional separation: application to

3 the isolation of multi-targeted compounds from Edelweiss plant

4 Léa Marlot ${ }^{1}$, Magali Batteau ${ }^{1}$, Karine Faure ${ }^{1}$

$5 \quad{ }^{1}$ Université de Lyon, CNRS, Université Claude Bernard Lyon 1, ENS de Lyon, Institut des Sciences

6 Analytiques, UMR 5280, 5 rue de la Doua, F-69100 VILLEURBANNE, France

7

8 Keywords

9 Preparative chromatography, comprehensive two-dimensional chromatography, centrifugal partition

10 chromatography, countercurrent chromatography 


\section{Abstract}

Preparative two-dimensional chromatography is gaining interest in the elucidation of complex samples as it allows the recovery of a large number of molecules without the risks inherent to tedious multi-step sample preparation. While the second dimension is often selected to be liquid chromatography, it may be of interest to compare the specificities of two different techniques, namely liquid chromatography and centrifugal partition chromatography, to be used as first

17 dimension.

A fair comparison between off-line CPCxLC and prepLCxLC in selective comprehensive mode for preparative purposes is carried out in this study, illustrated by the isolation of five compounds from high-value Edelweiss plant. The method development of each configuration is achieved on laboratory scale instruments. The quality of separation is compared using 2D-contour plots. The prepLCXLC exhibits a large separation space that leads to an overall large peak capacity, which is of great interest for complex samples. But its limited loading capacity involves a large number of ${ }^{2} D$ runs increasing the running costs for preparative purposes. On the other hand, CPCXLC provides a low peak capacity due to the poor efficiency provided by CPC. However, this liquid-liquid technique can be finely tuned to generate a high selectivity, decreasing the number of runs necessary to produce a limited number of target solutes. 


\section{Introduction}

In recent years, two-dimensional separations are becoming increasingly implemented in a wide range of applications as complex samples are more and more appealing. These 2D separations can be set up for analytical purposes in order to detect and identify new molecules [1, 2]. They can also be used for preparative purposes, to produce unknown target to be sent to further analysis such as NMR, IR or mass quantification, or to generate small amounts of reference substances from high value samples. In these preparative issues, it is crucial that the entire sample is subjected to the two dimensions without any loss. Thus, the implementation of 2D separation for preparative purposes has different constraints and requirements than the ones for analytical purposes.

In the literature, 2D preparative separations have just emerged, mostly in the natural product field [3-11] where the discovery of new active compounds is intrinsically related to the unraveling of the complex matrix. The techniques involved in the 2D setups are liquid chromatography (LC) and countercurrent chromatography (CCC), the later one using a liquid stationary phase. All combinations exist (LC/LC, LC/CCC, CCC/CCC), but the separations involving only countercurrent apparatus are very rarely implemented due to the low efficiency of the technique [9-12]. Amongst countercurrent technologies, centrifugal partition chromatography technique (CPC) is of particular interest as it can strongly hold liquid stationary phase at fast flow rate and high sample concentration.

In the literature, CPC and LC have already been compared in one-dimensional separation for preparative objectives $[13,14]$. The purpose of this publication is to fairly compare the advantages and drawbacks of each technique in the 2D configurations $C P C x L C$ and prepLCxLC, keeping the second dimension strictly identical throughout the study.

The 2D preparative separation was applied to the isolation of multi-targeted compounds from a rare sample with high yield and purity requirements. Edelweiss is a protected plant from European Alps which produces a wide diversity of secondary plant metabolites, especially some with anti-oxidative properties due to its growing at high altitude [15-17]. While the plant is just started to get cultivated mainly in Austria and Switzerland, it is essential to qualify the biological activity and chemical 
structures of its compounds of interest. Recently two main compounds, namely leontopodic acid A and 3,5-dicaffeoylquinic acid, have been isolated by the combination of CPC and LC in heart-cut mode, both at lab-scale and pilote scale [18]. In the present study, the two-dimensional preparative strategy is considered in a selective comprehensive mode [19] to recover five targeted compounds at laboratory scale (mg-scale).

\section{Materials and methods}

\subsection{Chemicals and materials}

All solvents were of analytical grade. Methyl tert-butyl ether was purchased from Acros Organics (Fisher Scientific, Illkirch, France). Formic acid, ammonium acetate and ethanol were purchased from Fisher Scientific. Acetonitrile was purchased from Biosolve Chimie (Dieuze, France). The dried aerial part of Edelweiss plant was provided by Extrasynthese (Genay, France).

\subsection{Instrumentation} The SpotPrep II system from Gilson (Saint-Avé, France) was set up as first dimension instrument, with a $5.2 \mathrm{ml}$ sample loop and detection set at $330 \mathrm{~nm}$. The CPC instrument was the FCPC-A frame from Kromaton Rousselet-Robatel (Annonay, France) equipped with a $35.8 \mathrm{ml}$ rotor and thermostated at $30^{\circ} \mathrm{C}$. The HPLC system used as second dimension was an Alliance 2690 system from Waters (SaintQuentin-en-Yvelines, France), set up at $330 \mathrm{~nm}$ and with a $100 \mu \mathrm{l}$ sample loop for the analytical step or $2.6 \mathrm{ml}$ sample loop for the loading step. The delay volume is $1 \mathrm{ml}$ without sample loop.

\subsection{Preparation of crude extract}

The crude extract was prepared from the dried aerial part of Edelweiss plant by macerating $4 \mathrm{~g}$ of the plant in $200 \mathrm{ml}$ of water/ethanol 70/30 (v/v) in acidic conditions for 16 hours. The mixture was centrifuged and the supernatant was passed through filter paper. Then the obtained sample was concentrated 10 times with the rotary evaporator to get $20 \mathrm{ml}$ of the crude extract in water. The final crude extract was filtrated through Nylon membrane $0.45 \mu \mathrm{m}$.

\subsection{First-dimension separations}


The CPC method was developed in our previous study [18]. A two-phase solvent system composed of methyl tert-butyl ether and water $\mathrm{pH} 3$ was prepared. The upper phase was equilibrated as stationary phase at $1720 \mathrm{rpm}$, while the lower phase as mobile phase was pumped into the column at a flow-rate of $5 \mathrm{ml} / \mathrm{min}$ in descending mode. When the equilibrium was established, $1 \mathrm{ml}$ of the crude extract was injected and the total run time was $50 \mathrm{~min}$. The fractions were collected every minute which corresponds to a recovery of 50 fractions of $5 \mathrm{ml}$.

For the prepLC method, a reversed-phase XSelect CSH Phenyl-Hexyl column (150 mm x 10 mm i.d. $5 \mu \mathrm{m}$, column dead volume $7.7 \mathrm{ml}$ ) was used as the stationary phase, thermostated at $30^{\circ} \mathrm{C}$. The mobile phase was composed of water at $10 \mathrm{mM}$ of ammonium acetate $(\mathrm{pH} 7)(\mathrm{A})$ and $\mathrm{ACN}(\mathrm{B})$ in linear gradient: $1 \%$ to $25 \%$ B for 19.2 minutes, $25 \%$ to $95 \%$ B for 0.8 minute, $95 \%$ to $1 \%$ B for 0.8 minute and $1 \%$ B for 4.2 minutes. The flow-rate was $9.5 \mathrm{ml} / \mathrm{min}$. After equilibration of the column, $1 \mathrm{ml}$ of the crude extract was injected for a total run time of 25 minutes. The fractions were collected every 0.5 minute which corresponds to a recovery of 50 fractions of $4.75 \mathrm{ml}$ each.

\subsection{Second-dimension LC separation}

A reversed-phase XSelect CSH Phenyl-Hexyl column (150 mm x $4.6 \mathrm{~mm}$ i.d. $5 \mu \mathrm{m}$, column dead volume $1.6 \mathrm{ml}$ ) was used as the second dimension HPLC column. For ${ }^{1} \mathrm{D}$ fraction analysis, an injection volume of $20 \mu \mathrm{l}$ ( $1 \%$ of the column volume) was selected. For the loading step, $65 \%$ of the ${ }^{2} \mathrm{D}$ column volume was injected meaning a fraction injection volume of $1 \mathrm{ml}$. The mobile phase was water with $0.1 \%$ of formic acid $(\mathrm{pH} 3)(\mathrm{A})$ and acetonitrile with $0.1 \%$ of formic acid $(\mathrm{B})$ in a gradient mode as following: $12 \%$ B for 1.1 minutes, $12 \%$ to $35 \%$ B for 18.4 minutes, $35 \%$ to $95 \%$ B for 0.8 minute, $95 \%$ to $12 \% \mathrm{~B}$ for 0.8 minute and $12 \% \mathrm{~B}$ for 3.9 minutes. The total elution time was 25 minutes. The flowrate of the mobile phase was $2 \mathrm{ml} / \mathrm{min}$ and the effluents were monitored at $330 \mathrm{~nm}$.

\section{Results and discussion}

\subsection{Comprehensive strategy implementation and resulting 2D-contour plots}


The HPLC chromatogram of the Edelweiss extract is shown in Figure 1. In this crude sample, the two compounds of interest from our previous study [18], namely 3,5-dicaffeoylquinic acid (compound A) and leontopodic acid A (compound D), are present in high quantity. Three other compounds, B, C and E, are unknown compounds that we suspect to be isomers and products of degradation of compound D. As shown on the chromatogram and in our previous study, the isolation of the five compounds of interest with high purity level cannot be considered by one-dimensional preparative LC or CPC due to insufficient resolution. Hence a two-dimensional preparative strategy is pursued. While many two-dimensional (2D) configurations exist for preparative purpose, it appears from our recent review [12] that using the highly resolutive LC technique in the second and last dimension provides better resolution and hence purification quality. Hence in this comparison, two preparative 2D configurations are compared (Figure 2). The first one is an off-line CPCxLC, using our previously developed CPC method, which has shown some interesting orthogonality degrees with RPLC methods. The second configuration is an off-line LCxLC configuration from which the orthogonality is obtained by changing the mobile phase $\mathrm{pH}$ while using the very same stationary phase. These configurations have been selected to be as cost-effective as possible, i.e. limiting column investment. For an understandable simplification, the first dimension is noted ${ }^{1} \mathrm{D}$ and the second is noted ${ }^{2} \mathrm{D}$. To get an estimation of the pros and cons of each first-dimension technology, the second dimension has been optimized and is similar in both configurations, i.e. based on phenyl hexyl stationary phase and acidic gradient mobile phase, as in Figure 1. This separation can be carried out on a semipreparative column ( $150 \mathrm{~mm} \times 10 \mathrm{~mm}$ i.d. $5 \mu \mathrm{m}$, column dead volume $7.7 \mathrm{ml}$ ) for the production step, but can also be scaled down to a laboratory-scale column $(150 \mathrm{~mm} \times 4.6 \mathrm{~mm}$ i.d. $5 \mu \mathrm{m}$, column dead volume $1.6 \mathrm{ml}$ ) for the development of our 2D strategy (Figure 2).

The off-line 2D separations are carried out, during production, in a selective comprehensive mode where only the fractions of interest from first dimension are sent to the second dimension (Figure 2, production step). This imposes that the ${ }^{1} D$ and ${ }^{2} D$ fractions are finely selected and that the fraction transfer between ${ }^{1} \mathrm{D}$ and ${ }^{2} \mathrm{D}$ columns is total without any split or loss. The method development of 
such a separation requires two steps in which the $2 \mathrm{D}$ separations are performed in a full

comprehensive mode with the second dimension implemented at smaller scale. In the first step, the orthogonality of the two dimensions is validated and/or optimized by monitoring the position of each compound of interest (Figure 2, analytical step). It requires only aliquots of the ${ }^{1} \mathrm{D}$ fractions to be analyzed, while in the second stage, the ${ }^{2} \mathrm{D}$ lab-scale column is loaded in such a way to simulate the full transfer of ${ }^{1} D$ fractions and hence ensures that the purity of the targeted compounds will not be compromised during the transfer process (Figure 2, loading step). It is important to notice here that the full transfer is processed without any treatment of the ${ }^{1} D$ fractions and this specification will remain during the study.

In order to conduct a fair comparison of the first-dimension technology, it is decided that the 2D configurations should initially treat the same number of fractions, as the overall cost of a 2D separation is mainly governed by the number of runs on the second dimension. In our previous study [18], the CPC eluent in first dimension was collected every minute in order to produce fractions volume of $5 \mathrm{ml}$. In the present study, this criterion is maintained which leads to the collection of 50 fractions from first dimension, and hence, $50^{2} \mathrm{D}$ runs for comprehensive separations. Moreover, the same sample load of $1 \mathrm{ml}$ is injected in ${ }^{1} \mathrm{D} C P C$ and prepLC to allow an equitable comparison. Of course, this initial injection could be further optimized but this is not the purpose of this publication.

\subsection{Quality evaluation of the $2 D$ separations at the analytical step}

With the objective to isolate as much targeted compounds as possible at the lowest cost, it is important to visualize the distribution of the targeted peaks regarding the others in the given separation space, because the distances between peaks usually relate to the loading capacity of a preparative method [20], providing that practical transfer conditions are favorable. Hence, the ${ }^{1} D$ separations, either CPC or prepLC, were performed and only $20-\mu$ l aliquots of the 50 recovered fractions were transferred in the ${ }^{2} \mathrm{D}$ HPLC separation. The resulting 2D-contour plots are shown in Figure 3. This visualization allows the evaluation of the peak distribution through two criteria: the 

separation, and the selectivity which describes the distribution of the peaks in the separation space.

\subsubsection{Peak distribution of prepLCXLC separation}

160

The sample peak capacity, noted $\mathrm{n}_{\mathrm{c}}$, was introduced in 1967 by Giddings [21] in order to describe the maximal number of peaks that could theoretically be separated by a given column. This notion was established for isocratic and gradient elution modes in one-dimensional separations [21-23]. In our prepLCXLC separation, the first and the second dimensions were conducted in gradient mode. The calculation of the sample peak capacity in gradient mode is provided by the following equation [22]:

$$
n_{c}=1+\frac{2.3 S \Delta C}{2.3 S s+1} \cdot \frac{\sqrt{N}}{4}
$$

With $\mathrm{S}$ the slope of the retention model of the compound (usually equal to 4 for small molecules), $\Delta C$ the elution composition range of the compounds, $\mathrm{s}$ the normalized gradient slope, and $\mathrm{N}$ the number of theoretical plates during the separation.

By implementing the ${ }^{1} \mathrm{D}$ prepLC in one-dimensional separation, the resulting sample peak capacity is 30 (with an efficiency of 4000 plates and an elution composition range of 0.24 ). Similarly, the resulting sample peak capacity of the ${ }^{2} \mathrm{D} L C$ implemented in one-dimensional separation is 30 due to the same efficiency and elution composition range.

The 2D theoretical sample peak capacity being the product of sample peak capacities in each dimension [24], its value for prepLCxLC is here 900 . This may allow the separation of around 300 compounds, as stated by Davis [25] who claim that the effective peak number that can be separated is $37 \%$ of the theoretical peak capacity. On the Figure 3a corresponding to the $2 \mathrm{D}$-contour plot of the prepLCxLC separation, about seventy compounds are separated.

The space occupation of the separation space is high with a value of $69 \%$ (calculated using Convex Hull method [26]). Despite this large space occupation, no peak elutes at bottom right corner and top left corner of the 2D-contour plot. Indeed, reversed phase chromatography mechanisms involving hydrophobic interactions between compounds and stationary phase are implemented in both dimensions. Consequently, the same type of interactions is settled in the two dimensions and thus, 

despite the $\mathrm{pH}$ switch (from $\mathrm{pH} 7$ to $\mathrm{pH} 3$ ) between dimensions, the retention data are correlated leading to a peak distribution along a regression line with a certain degree of dispersion around this line. Indeed, to reduce the costs of investment, we experimented here an orthogonality implementation based only on the change of ionization state of solutes towards the very same reversed phase stationary phase. The selectivity based on the electronic properties of the solutes is of interest for natural products, where a large number of organic acids and bases can be found. Compounds that remain in the same ionization state during the $\mathrm{pH}$ switch are to be found on the regression line $\mathrm{x}=\mathrm{y}$ on the $2 \mathrm{D}$-contour plot. This is the case for compound $\mathrm{B}$, which provides an extra information on this unknown compound. Because hydrophobic interactions are decreased when the solute is ionized, tracking the solutes during this $\mathrm{pH}$ switch through their position on the 2D plot provides an insight of their pKa. For example, known compound A (3,5-dicaffeoylquinic acid) is less retained in the second dimension than in the first one, indicating that it is more ionized at $\mathrm{pH} 3$. This is coherent with its known pKa of 3.3. Conversely, leontopodic acid A (compound D) and unknown compounds $\mathrm{C}$ and $\mathrm{E}$ are less retained in the first dimension than in the second one thus are more ionized at $\mathrm{pH} 7$ than at $\mathrm{pH} 3$.

The prepLCxLC separation with a high theoretical sample peak capacity allows the spread of the whole matrix in the separation space, and an homogeneous distribution of compounds, at the detriment of selectivity.

\subsubsection{Peak distribution of $C P C x L C$ separation}

In our application, the CPC elution was conducted in isocratic mode. This has a consequence on the sample peak capacity, as in isocratic mode, peaks become broader as they are more retained. The sample peak capacity in isocratic mode is provided by the following equation [21, 24]:

$$
n_{c}=\frac{\sqrt{N}}{4} \ln \left(\frac{V r_{n}}{V r_{1}}\right)
$$


With $\mathrm{N}$ the number of theoretical plates during the separation, $\mathrm{V} r_{1}$ and $V r_{n}$ the retention volume of the first and the last retained compound respectively.

With an efficiency less than 500 plates and a retention volume window from 20 to $220 \mathrm{ml}$ (Figure 3b), the sample peak capacity in ${ }^{1} \mathrm{D}$ CPC is around 10 peaks. The low efficiency of the CPC can be observed on the Figure $3 \mathrm{~b}$ where peaks are spread out along the CPC axis. This well-known lower efficiency is due to the use of a liquid stationary phase and its consequent slow mass transfer. As calculated previously, the sample peak capacity of the ${ }^{2} D$ LC is 30 . Thus, the theoretical sample peak capacity of the CPCXLC separation is around 300 meaning that the number of peaks that can be separated in the resulting separation space is three times lower than with the previous LCXLC method. Of course, this theoretical comparison does not take non-ideal transfer due to injection effects into account (see below practical condition assessment).

As shown on the Figure $3 \mathrm{~b}$ corresponding to the $2 \mathrm{D}$-contour plot of the $\mathrm{CPCxLC}$ separation, the important baseline width of the peaks does not allow the separation of the whole matrix in the separation space. So the strategy to separate the targeted compounds from the rest of the matrix must rely on an important selectivity between them. The CPC separation is based on the partition of the solute between two liquid phases and hence can rely on several interactions such as Van der Waals, hydrogen or hydrophobic bonds depending on the solvent system. Thus, the choice of the solvents (nature and composition) that composed the stationary and mobile phases is important in CPC in order to involve the expected interactions. In our example, methyl tert-butyl ether was chosen as stationary phase with acidified water as mobile phase. This reversed-phase column can involve hydrophobic interactions, hydrogen bonds as well as Van der Waals bonds with the targeted compounds (K values over 3 ). As the ${ }^{2} \mathrm{D} L \mathrm{C}$ relies only on hydrophobic interactions, the retention data from the first and second dimension separations can be non correlated offering a high selectivity between peaks and a high occupation of the separation space as observed on the 2D-contour plot. One of the advantages of CPC is the large choice of the solvent systems allowing a refined selection of the solvent system specifically adapted to the desired separation. This high selectivity is an 
obvious requirement in order to compensate for the low efficiency of CPC. The implementation of LC as second dimension brings more efficiency to finally isolate targeted compounds.

\subsubsection{Practical condition assessment}

As explained in Stoll et al. and Dugo et al. reviews in 2007 and 2008 [1, 27], the implementation of comprehensive 2D separations allows an improvement in resolving power by increasing the effective separation, the fractionation of the ${ }^{1} \mathrm{D}$ eluent and the transfer of the fractions to the second dimension are of great concern $[1,24,27]$.

The ${ }^{1} \mathrm{D}$ eluent has to be fractionated in a strategic way in order to preserve the ${ }^{1} \mathrm{D}$ separation during the transfer to the second dimension. This fractionation can be evaluated through the ${ }^{1} \mathrm{D}$ undersampling factor noted $\alpha[29,30]$ :

$$
\alpha=\frac{1}{\sqrt{1+0.21\left(\frac{t_{s}}{{ }^{1} \sigma_{t, o b s}}\right)^{2}}}
$$

With $t_{s}$ the sampling time of the first-dimension eluent and ${ }^{1} \sigma_{t, o b s}$ the ${ }^{1} D$ peak width.

To preserve the ${ }^{1} \mathrm{D}$ separation to the second dimension, the factor $\alpha$ must be at least 0.8 meaning that each peak from first dimension is cut at least in three fractions. If this criterion is not respected, separated peaks in first dimension may re-mix during transfer leading to a decrease of the sample peak capacity and a doubt concerning the purity of the compounds $[28,31]$. For the prepLCxLC separation, the baseline width of the ${ }^{1} \mathrm{D}$ peaks is around $1.5 \mathrm{~min}$. Thus, with a sampling time of 0.5 min, the peaks are adequately cut in three fractions. For the CPCXLC separation, as the CPC was implemented in isocratic elution, the width of the peaks becomes broader with the retention. For the targeted compounds, the smallest baseline width is $15 \mathrm{~min}$ (compound E). Thus, with a sampling time of $1 \mathrm{~min}$, the undersampling is not an issue with an $\alpha$ value of 0.98 . For a larger fractionation of peaks in three cuts $(\alpha=0.8)$, the sampling time should not exceed $5 \mathrm{~min}$. Thus, with the implementation of 
CPC in first dimension, the sampling time can be 10 times higher than for prepLC, which induces less fractions to inject on second dimension for a comprehensive strategy.

As explained in François et al. review in 2009 [24], the solvents compatibility between the ${ }^{1}$ D eluent contained in transferred fractions and the ${ }^{2} \mathrm{D}$ mobile phase is another major issue. In order to maintain the ${ }^{2} \mathrm{D}$ resolution, it is of importance to avoid peaks widening during the ${ }^{2} \mathrm{D}$ injection. These negative effects can be due to the absence of focusing effect [32] or due to viscous fingering [33, 34]. In off-line process, an intermediate treatment of the fractions can avoid these effects. This is however really tedious and time consuming when many fractions are to be transferred, which is usually the case in any 2D preparative strategy. In analytical 2D LC, the issue is avoided by transferring small amounts or by using trapping columns $[35,36]$. With 2D preparative aim, however, the volumes to be treated are usually not compatible with this kind of solution. If no fraction treatment is carried out, these peak band broadening due to injection effects can be evaluated through the correction factor noted $\beta$ [29]:

$$
\beta=\frac{1}{\sqrt{1+\frac{1}{\delta i^{2}} \cdot \frac{{ }^{2} V i^{2}}{{ }^{2}{\sigma_{v, \text { col }}}^{2}} \cdot \frac{1}{{C_{F}}^{2}}}}
$$

With $\delta \mathrm{i}^{2}$ a parameter related to the shape of the injection band (equal to 4 in a practical case), ${ }^{2} \mathrm{Vi}$ the ${ }^{2} \mathrm{D}$ injection volume, ${ }^{2} \sigma_{\mathrm{v}, \mathrm{col}}{ }^{2}$ the standard deviation resulting from the column dispersion and $\mathrm{C}_{\mathrm{F}}$ the compression factor.

The injection effects on second dimension are favorable when the factor $\beta$ is close to 1 . In our comparison of $C P C X L C$ and prepLCxLC separations, with a same transferred volume ${ }^{2} \mathrm{Vi}$ on the same ${ }^{2} D$ column $\left({ }^{2} \sigma_{v, c o l}{ }^{2}\right)$, the ${ }^{2} D$ injection effect factor $\beta$ only depends on the compression factor $C_{F}[37$, 38]. This one is related to the solute retention factors on the second dimension, and defined as:

$$
C_{F}=\frac{1+{ }^{2} k_{e, 1}}{1+{ }^{2} k_{e, 2}}
$$

With ${ }^{2} k_{e, 1}$ the solute retention factor on the ${ }^{2} \mathrm{D}$ column, in ${ }^{1} \mathrm{D}$ mobile phase at elution (fraction solvent) and ${ }^{2} k_{e, 2}$ the solute retention factor on the ${ }^{2} \mathrm{D}$ column in ${ }^{2} \mathrm{D}$ mobile phase at elution. 
The compression factor is favorable $(>1)$ if the injection solvent in the transferred fraction has a weaker eluent strength than the mobile phase at peak elution in second dimension. As the second dimension shows a reverse-phase mechanism and runs with a mobile phase composed of $88 \%$ water to $65 \%$ water during the elution (with acetonitrile as organic solvent), the solvent in fractions must contain more water than the mobile phase at elution. For the prepLC implemented as first dimension, the solvent in fractions was composed from $99 \%$ water for the first fraction to $75 \%$ water for the last fraction. In this case, the compression factor is higher than 1 for only a part of the peaks. The part where the compression factor is lower than 1 can be visualized on the top left corner of the 2D-contour plot on the Figure 3a where the eluent strength of the injection solvent (solvent in fraction) is stronger than the eluent strength of the mobile phase. Thus, for this part, there is no focusing effect on the peaks and some deformation can occur. On the 2D-contour plot, no negative effects are observed for the analytical step due to low ${ }^{2} D$ injection volume but these effects are visible during the loading step with higher ${ }^{2} \mathrm{D}$ injection volume (Figure $3 \mathrm{c}$ ). When $\mathrm{CPC}$ was implemented as first dimension, and run in isocratic mode, all ${ }^{1} \mathrm{D}$ fractions are composed of $93.5 \%$ water (with MtBE as organic solvent), which is obviously favorable going into a $88 \%-65 \%{ }^{2} \mathrm{D}$ gradient elution. These conditions give a compression factor much higher than 1 for all fractions separations allowing a focusing effect on all peaks. We found that the compatibility of solvent between both dimensions is easier to deal with when an isocratic tunable mobile phase is used in first dimension, and CPC technique may offer that opportunity. It is important to notice here that the dilution factor $D_{F}$ is higher for the $C P C x L C$ separation which can lead to some detection problems. As described in the following equation $[39,40]$, the dilution factor depends on the peak width observed on first-dimension and the compression factor. For CPCxLC, despite a high compression factor during transfer, the dramatically low efficiency of the CPC technique leads to a large peak width hence a high dilution factor in the order of 75 , in opposition to 


$$
D_{F}=\frac{\sqrt{2 \pi}}{{ }^{1} V i} \cdot{ }^{1} \sigma_{v, o b s} \cdot \frac{\sqrt{2 \pi}}{\delta i \sqrt{1-\beta^{2}}} \cdot \frac{1}{C_{F}}
$$

Where $\delta^{2} i$ is a parameter dependent on the injection process, ${ }^{1} \sigma v$, col is the standard deviation resulting of band broadening in the ${ }^{1} \mathrm{D}$ column and ${ }^{1} \mathrm{Vi}$ is the injected volume in $1 \mathrm{D}$.

\subsection{Quality evaluation of the $2 D$ separations when loading}

Since scale-up is easily achieved on both techniques, the requirement before moving to production is the overloading study. As the objective is the full recovery of compounds of interest, the injection volume in second dimension has to be equal to the fraction volume obtained from first dimension. In our study, the fraction volume from each first dimensions is initially $5 \mathrm{ml}$. Transferring this amount to a $10 \mathrm{~mm}$ internal diameter prepLC column represents an injection volume of $65 \%$ of its dead volume.

This loading study can be evaluated on the second dimension with a smaller $4.6 \mathrm{~mm}$ internal diameter column (specifications in material and methods section). So, the study of such a volume load on a $4.6 \mathrm{~mm}$ internal diameter column can be achieved by injecting $65 \%$ of its volume, i.e. $1 \mathrm{ml}$. The 2D-contour plots resulting from the loading step are shown in Figures $3 \mathrm{c}$ and $3 \mathrm{~d}$.

\subsubsection{Resulting peaks resolution on second dimension}

Some peaks deformations can be observed on the top left corner of the 2D contour plot of the prepLCXLC separation (Figure 3c). As already mentioned, this is due to the decrease of the ${ }^{2} \mathrm{D}$ volume, the ratio ${ }^{2} \mathrm{Vi}^{2} /{ }^{2} \sigma_{\mathrm{v}, \mathrm{col}}{ }^{2}$ is 2500 times higher than during the analytical step. These effects do not appear with the use of ${ }^{1} \mathrm{D} C P C$ due to the much higher compression factor that compensates for the larger transferred fraction volume.

The resolution loss after the $65 \%$ load can be visualized on critical pair of targeted compounds (blue circles, Figure 3). For prepLCxLC, the critical pair is compound $D$ with its impurity $i_{D}$ contained in fraction \#30 (from 14.5 to 15 minutes) and for CPCXLC, the critical pair is compounds A and B 
contained in fraction \#30 (from 29 to 30 minutes). The chromatograms of both fractions \#30 from prepLC and CPC are shown on Figure 4.

It can be seen that the resolution is totally lost between peaks $D$ and $i_{D}$ from ${ }^{1} D$ prepLC $\# 30$ when the

${ }^{2} \mathrm{D}$ LC column is loaded in preparative conditions (Figure 4a and c). Hence with these loading specifications, corresponding to $5 \mathrm{ml}$ fractions being fully transferred to a $10 \mathrm{~mm}$ internal diameter ${ }^{2} \mathrm{D}$ column, only four compounds can be isolated as shown on Figure $3 c$. The compound $D$ has coeluted with an impurity. Conversely, peaks A and B from CPC \#30 keep the same resolution during loading and the ${ }^{2} \mathrm{D} L C$ column is not saturated (Figure $4 b$ and d). This is due to the high dilution of the compounds during CPC and the high compression factor of the peaks during the transfer to the second dimension, as mentioned before. Because the solutes coming from CPC are highly diluted in a low strength solvent, a $65 \%$ injection volume is easily handled by the ${ }^{2} \mathrm{D}$ column. The overall $2 \mathrm{D}$ plot (Figure 3d) shows that the five targeted compounds can be fully transferred and isolated with baseline return.

It is clear through this study that the solvent compatibility issue is even more problematic when injection large volumes of fractions on the second dimension. The selections of the first dimension and its elution mobile phase composition are crucial in the resulting preparative performances. Because ${ }^{1} \mathrm{D}$ prepLC and CPC have here very different elution phases, it is interesting to optimize the transferred volume for each configuration.

\subsubsection{Charge capacity on second dimension for prepLCXLC separation}

The injection volume of the critical fraction $\# 30$ from ${ }^{1} \mathrm{D}$ prepLC was incrementally increased to achieve baseline return between peak $D$ and its impurity. For the quantitative evaluation of the return to baseline [41], the free-space between peaks, noted $\Delta \mathrm{V}$ (expressed in $\mathrm{ml}$ ), was calculated with the following equation:

$$
\Delta V=\left(V_{r, 2}-V_{r, 1}\right)-3\left(\sigma_{v, 1}+\sigma_{v, 2}\right)
$$


With the retention volumes of peaks 1 and 2 and their corresponding standard deviation in volume unit. As the peaks are not symmetric with the load, the standard deviations were calculating from the right peak width for peak 1 and left peak width for peak 2 . Statistically, $99.7 \%$ of the solute molecules can be found within the 6- $\sigma$ baseline width which was a satisfactory criterion for the evaluation of the baseline return. Thus, using the ${ }^{1} \mathrm{D}$ prepLC $\# 30$ fraction as sample, the $\Delta V$ was calculated for the injection volume of $1 \%, 6 \%, 13 \%, 19 \%, 25 \%, 31 \%$ and $65 \%{ }^{2} \mathrm{D}$ LC column. In our study, the maximum load is observed for an injection volume of $19 \%$.

If the specifications require that the fraction coming from the first dimension is fully injected in the second one, the use of a $150 \times 10 \mathrm{~mm}$ i.d. prepLC as ${ }^{2} \mathrm{D}$ imposes that the ${ }^{1} \mathrm{D}$ fractions need to be reduced down to $1.5 \mathrm{ml}$ each. It then represents a sampling time of $0.16 \mathrm{~min}$ on the ${ }^{1} \mathrm{D}$ prepLC and the collection of 157 fractions. In this case, as the sampling time is much lower than 0.5 minute, the peaks from first dimension are cut in more than three fractions leading to the ${ }^{1} D$ undersampling factor $\alpha$ closer to 1 and thus favorable for the separation. Concerning the ${ }^{2} D$ injection effect factor $\beta$, as the injection volume on the second dimension decreases from $65 \%$ to $19 \%$ of the column dead volume, the ratio ${ }^{2} \mathrm{Vi}^{2} /{ }^{2} \sigma_{\mathrm{v}, \text { col }}{ }^{2}$ will be 11 times minimized leading to factor closer to 1 and thus the negative effects will be more negligible. Injecting $19 \%$ of the column dead volume on a $150 \times 4.6 \mathrm{~mm}$ i.d. column or on a $150 \times 10 \mathrm{~mm}$ i.d. prepLC column, the ratio ${ }^{2} \mathrm{Vi}^{2} /{ }^{2} \sigma_{\mathrm{v}, \text { col }}{ }^{2}$ remains the same and so the ${ }^{2} D$ injection effect factor $\beta$. Another solution, to avoid the huge running cost of $157^{2} \mathrm{D}$ runs is to increase the size of the ${ }^{2} \mathrm{D}$ column to decrease the number of ${ }^{1} \mathrm{D}$ fractions. The maximal fraction volume that can be collected while keep reasonable ${ }^{1} \mathrm{D}$ undersampling factor $\alpha$ is $4.75 \mathrm{ml}$. To handle such an injection volume without overloading effects, a prepLC column with an internal diameter of $19 \mathrm{~mm}$ is necessary. Hence, 50 fractions of $4.75 \mathrm{ml}$ can be transferred leading to a lower $\alpha$ factor and an unmodified $\beta$ 379 factor.

\subsubsection{Charge capacity on second dimension for CPCXLC separation}


It was previously found that injecting ${ }^{1} \mathrm{D} C P C$ fraction to the extent of $65 \%{ }^{2} \mathrm{D}$ column was easily achieved without any resolution loss. Increasing the transferred volume, we noticed that it was possible to inject the entire CPC fractions of $5 \mathrm{ml}$ on the analytical LC column ( $150 \times 4.6 \mathrm{~mm}$ i.d.) meaning $313 \%$ of the LC column dead volume without any resolution loss.

Using the prepLC column ( $150 \times 10 \mathrm{~mm}$ i.d.) as second dimension, 24-ml fractions could then be injected on the second dimension. This is obtained by increasing the ${ }^{1} \mathrm{D}$ sampling time to $4.8 \mathrm{~min}$, which results in the collection of only 11 fractions from first dimension. In this case, the sampling time is maximal leading to a ${ }^{1} \mathrm{D}$ undersampling factor $\alpha$ of 0.8 lower than previously but still very favorable for the separation. Concerning the ${ }^{2} D$ injection effect factor $\beta$, the injection volume increases from $65 \%$ to $313 \%$ of the column dead volume leading to a 25 times increase of the ratio ${ }^{2} \mathrm{Vi}^{2} /{ }^{2} \sigma_{\mathrm{v}, \text { col }}{ }^{2}$ and so a lower $\beta$ factor than previously but as the compression factor is very important, the resolution is kept.

\subsection{Final 2D separations comparison}

In order to fairly compare off-line CPCxLC and prepLCxLC selective comprehensive separations based on the purification of $1 \mathrm{ml}$ rare Edelweiss extract, the various optimized options are here compiled together with solvent consumption and duration in Table 1.

The ${ }^{1} \mathrm{D}$ prepLC $(150 \times 10 \mathrm{~mm}$ i.d.) requires $475 \mathrm{ml}$ of solvent for a total duration of 50 minutes (including blank run). For the implementation of CPC in first dimension, a total volume of $325 \mathrm{ml}$ of solvent was used (mobile and stationary phases included) for a total time of 65 minutes (including equilibration and separation time). With the implementation of a ${ }^{2} \mathrm{D}$ prepLC column with the very same dimensions (no supplementary investment), 157 fractions of $1.5 \mathrm{ml}$ can be collected from ${ }^{1} \mathrm{D}$ prepLC. Using a selective comprehensive mode to isolate only the five targets, the injection of 29 fractions on second dimension is necessary with total time of 775 minutes. 
In the case of CPCXLC separation, 11 fractions of $24 \mathrm{ml}$ can be collected from ${ }^{1} \mathrm{D} C P C$ to be sent on the prepLC column (150 x $10 \mathrm{~mm}$ i.d.). In selective comprehensive mode, this combination requires the injection of only 7 fractions on second dimension for a total time of 240 minutes. In order to minimize the second-dimension investment cost, alternatives can be implemented. For the prepLCxLC separation, a higher internal diameter ${ }^{2} D$ column (column $150 \times 19 \mathrm{~mm}$ i.d.) can be implemented allowing 3.3 times less fractions to be injected on the ${ }^{2} \mathrm{D}$ column. Additional investment costs have to be considered. For the $\mathrm{CPCxLC}$ separation, the ${ }^{2} \mathrm{D}$ column used during method development (column $150 \times 4.6 \mathrm{~mm}$ i.d.) can be implemented for preparative purposes leading to 4.8 times more fractions to be injected on second dimension but with no need to purchase a prepLC All these options can be implemented with favorable practical conditions ( $\alpha$ and $\beta$ factors). If less fractions are to be collected and injected on ${ }^{2} \mathrm{D}$ column in order to further minimize the seconddimension running costs, a heart-cut mode can be implemented. In this case, the ${ }^{1} \mathrm{D}$ undersampling factor $\alpha$ and the ${ }^{2} D$ injection effect factor $\beta$ would not be favorable anymore leading eventually to a re-mix of the compounds from first-dimension separation and peaks deformation during the transfer and thus an important loss of the resolution between peaks. Other alternatives to either reduce the fraction volume or increase the compression factor by make-up addition would be of interest but may greatly increase the overall separation process duration.

\section{Conclusion}

This study represents the first comparison between CPC and prepLC used as first dimension in an offline two-dimensional separation. The two-dimensional CPCXLC and prepLCXLC separations were performed in comprehensive mode at lab-scale in order to develop the selective comprehensive 2D separations for the isolation of five targeted compounds from Edelweiss plant. As the investment cost on second dimension was initially stated for both separations, the first dimensions CPC and prepLC performances could be compared. 
While the conventional LCXLC setup demonstrates once again its powerful peak capacity and resolution, it requires a large investment and exhibits solvent compatibility issues. The CPC technique may be an appropriate alternative when dealing with these solvent compatibility issues since the

434 choice of the chemical nature of the CPC columns is much wider and can, if finely tuned, be of great

435 interest for preparative purposes. In both cases, CPC and prepLC are attractive techniques with

436 advantages and drawbacks, offering a great deal of possibilities for the development of 2D

437 preparative separations for rare samples.

438

439 Acknowledgment

440 The authors wish to deeply thank Extrasynthese company for providing the aerial part of Edelweiss 441 plant. 
443 [1] Dugo, P., Cacciola, F., Kumm, T., Dugo, G., Mondello, L., Journal of Chromatography A 2008, 1184, 353-368.

[2] Iguiniz, M., Heinisch, S., Journal of Pharmaceutical and Biomedical Analysis 2017, 145, 482-503. [3] Qiu, Y.-K., Chen, F.-F., Zhang, L.-L., Yan, X., Chen, L., Fang, M.-J., Wu, Z., Analytica Chimica Acta 2014, 820, 176-186.

448 [4] Wang, X.-Y., Li, J.-F., Jian, Y.-M., Wu, Z., Fang, M.-J., Qiu, Y.-K., Journal of Chromatography A 2015, 1387, 60-68.

[5] Li, J.-F., Fang, H., Yan, X., Chang, F.-R., Wu, Z., Wu, Y.-L., Qiu, Y.-K., Journal of Chromatography A 2016, 1456, 169-175.

[6] Qiu, Y.-K., Yan, X., Fang, M.-J., Chen, L., Wu, Z., Zhao, Y.-F., Journal of Chromatography A 2014, 1331, 80-89.

[7] Xie, X.-M., Sun, W.-Y., Huang, J.-Y., Polachi, N., Tong, L., Sun, G.-X., Chinese Journal of Analytical Chemistry 2016, 44, 1140-1147.

[8] Yan, X., Wang, L.-J., Wu, Z., Wu, Y.-L., Liu, X.-X., Chang, F.-R., Fang, M.-J., Qiu, Y.-K., Journal of Chromatography B 2016, 1033-1034, 1-8.

[9] Su, W., Liu, Q., Yang, Q., Yu, J., Chen, X., Journal of Separation Science 2013, 36, 3338-3344. [10] Liu, Q., Shi, S., Liu, L., Yang, H., Su, W., Chen, X., Journal of Chromatography A 2013, 1304, $183-$ 193.

[11] Jiang, S., Liu, Q., Xie, Y., Zeng, H., Zhang, L., Jiang, X., Chen, X., Food Chemistry 2015, 186, 153159.

[12] Marlot, L., Faure, K., Journal of Chromatography A 2017, 1494, 1-17.

[13] DeAmicis, C., Edwards, N. A., Giles, M. B., Harris, G. H., Hewitson, P., Janaway, L., Ignatova, S., Journal of Chromatography A 2011, 1218, 6122-6127.

[14] Li, S., Wang, W., Tang, H., Chen, K., Yang, J., He, L., Ye, H., Peng, A., Chen, L., Journal of Chromatography A 2014, 1344, 51-58.

468 [15] Dobner, M. J., Schwaiger, S., Jenewein, I. H., Stuppner, H., Journal of Ethnopharmacology 2003, 469 89, 301-303. Dobner, M. J., Sosa, S., Schwaiger, S., Altinier, G., Della Loggia, R., Kaneider, N. C., Stuppner, H., Planta medica 2004, 70, 502-508.

[17] Schwaiger, S., Cervellati, R., Seger, C., Ellmerer, E. P., About, N., Renimel, I., Godenir, C., André, P., Gafner, F., Stuppner, H., Tetrahedron 2005, 61, 4621-4630.

[18] Marlot, L., Batteau, M., Escofet, M.-C., Nuccio, S., Coquoin, V., De Vaumas, R., Faure, K., Journal of Chromatography A 2017, 1504, 55-63.

[19] Groskreutz, S. R., Swenson, M. M., Secor, L. B., Stoll, D. R., Journal of Chromatography A 2012, 1228, 31-40.

[20] Golshan-Shirazi, S., Guiochon, G., Journal of Chromatography A 1991, 536, 57-73.

[21] Giddings, J. C., Analytical Chemistry 1967, 39, 1027-1028.

[22] D’Attoma, A., Grivel, C., Heinisch, S., Journal of Chromatography A 2012, 1262, 148-159.

[23] Snyder, L. R., Dolan, J. W., Gant, J. R., Journal of Chromatography A 1979, 165, 3-30.

[24] François, I., Sandra, K., Sandra, P., Analytica Chimica Acta 2009, 641, 14-31.

[25] Davis, J. M., Giddings, J. C., Analytical Chemistry 1983, 55, 418-424.

[26] Semard, G., Peulon-Agasse, V., Bruchet, A., Bouillon, J.-P., Cardinaël, P., Journal of Chromatography A 2010, 1217, 5449-5454.

[27] Stoll, D. R., Li, X., Wang, X., Carr, P. W., Porter, S. E. G., Rutan, S. C., Journal of Chromatography A 2007, 1168, 3-43.

[28] Murphy, R. E., Schure, M. R., Foley, J. P., Analytical Chemistry 1998, 70, 1585-1594.

[29] Bedani, F., Schoenmakers, P. J., Janssen, H.-G., Journal of Separation Science 2012, 35, 1697 1711.

[30] Davis, J. M., Stoll, D. R., Carr, P. W., Analytical Chemistry 2008, 80, 461-473.

[31] Seeley, J. V., Journal of Chromatography A 2002, 962, 21-27. 
496 [34] Keunchkarian, S., Reta, M., Romero, L., Castells, C., Journal of Chromatography A 2006, 1119, 2049728.

498 [35] Sommella, E., Ismail, O. H., Pagano, F., Pepe, G., Ostacolo, C., Mazzoccanti, G., Russo, M., Novellino, E., Gasparrini, F., Campiglia, P., Journal of Separation Science 2017, 40, 2188-2197. [36] Gargano, A. F. G., Duffin, M., Navarro, P., Schoenmakers, P. J., Analytical Chemistry 2016, 88, 1785-1793.

502 [37] Jandera, P., Česla, P., Hájek, T., Vohralík, G., Vyňuchalová, K., Fischer, J., Journal of Chromatography A 2008, 1189, 207-220. [38] Vivó-Truyols, G., van der Wal, S., Schoenmakers, P. J., Analytical Chemistry 2010, 82, 8525-8536. [39] Schure, M. R., Analytical Chemistry 1999, 71, 1645-1657. [41] Seidel-Morgenstern, A., Schulte, M., Epping, A., Preparative Chromatography, Wiley-VCH Verlag GmbH \& Co. KGaA 2012, pp. 7-46. 


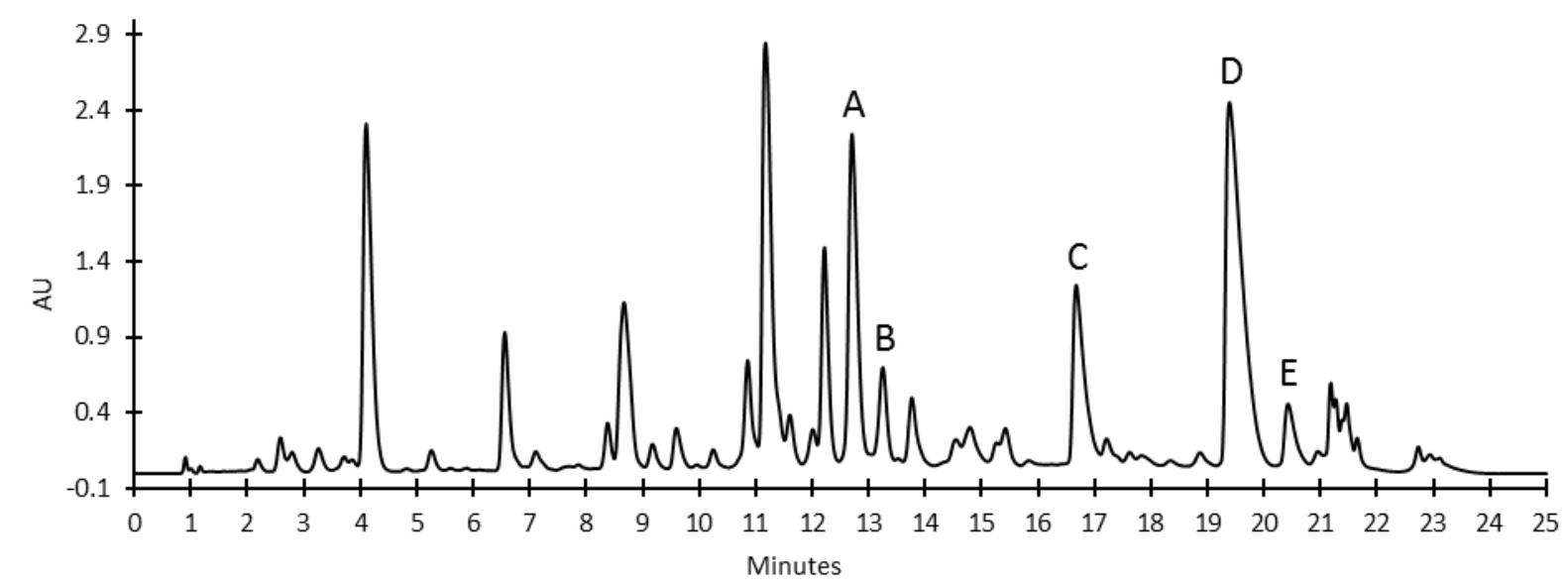

510 Figure 1. HPLC analysis of the crude sample on Phenyl-Hexyl column (150 mm x $4.6 \mathrm{~mm}$ i.d., $5 \mu \mathrm{m}$ ),

511 mobile phase of water $+0.1 \%$ of formic acid (A) and acetonitrile $+0.1 \%$ of formic acid (B) in gradient

512 mode: $12 \%$ B for $1.1 \mathrm{~min}, 12$ to $35 \% \mathrm{~B}$ in $18.4 \mathrm{~min}, 35$ to $95 \%$ B for $0.8 \mathrm{~min}$, flow-rate $2 \mathrm{ml} / \mathrm{min}$,

513 detection $330 \mathrm{~nm}$. 
$1^{\text {st }}$ dimensions

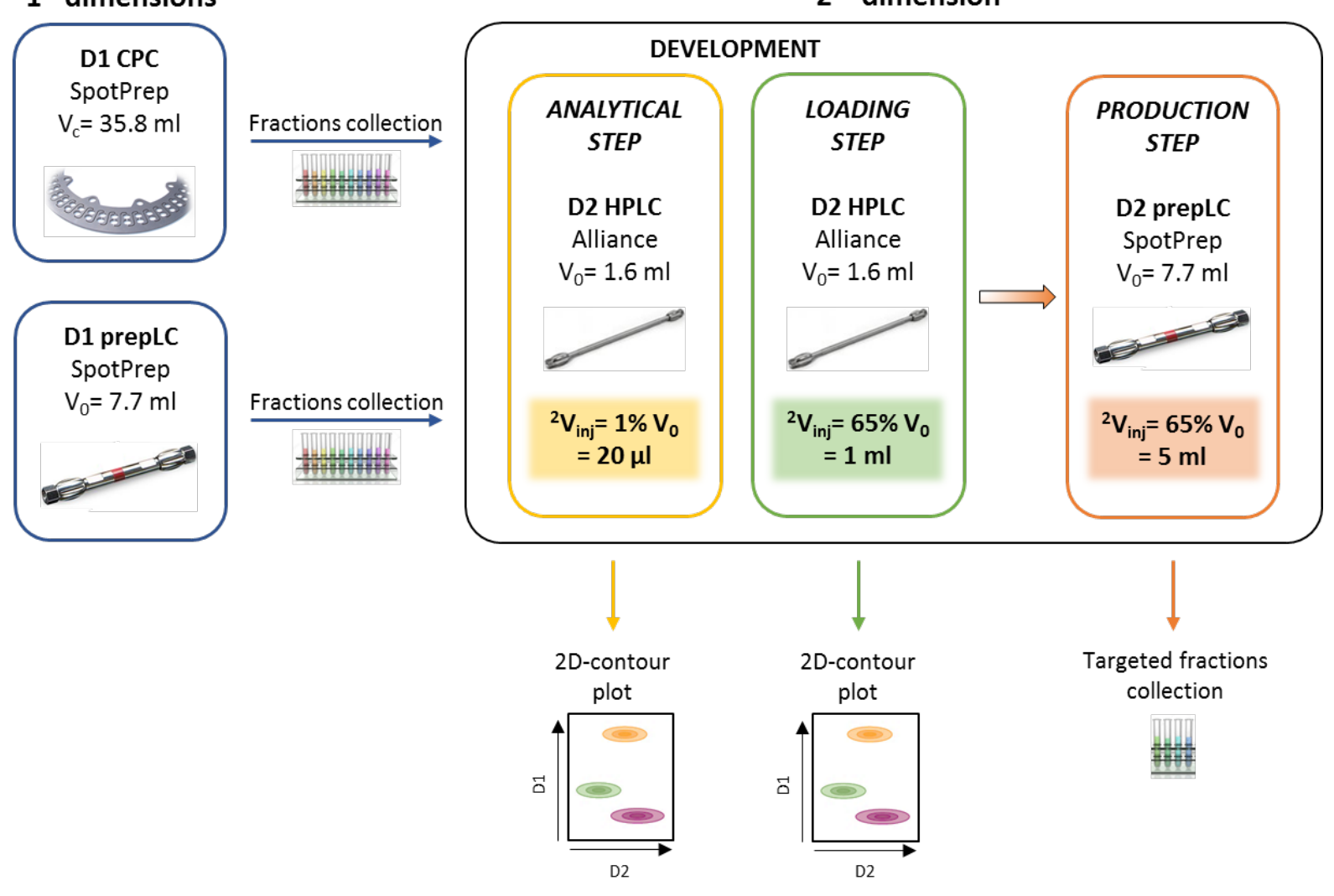

515 Figure 2. Two-dimensional process with CPC and prepLC as first dimensions and LC as second

516 dimension with the various sizes corresponding to the successive development steps. 
(a) prepLCxLC

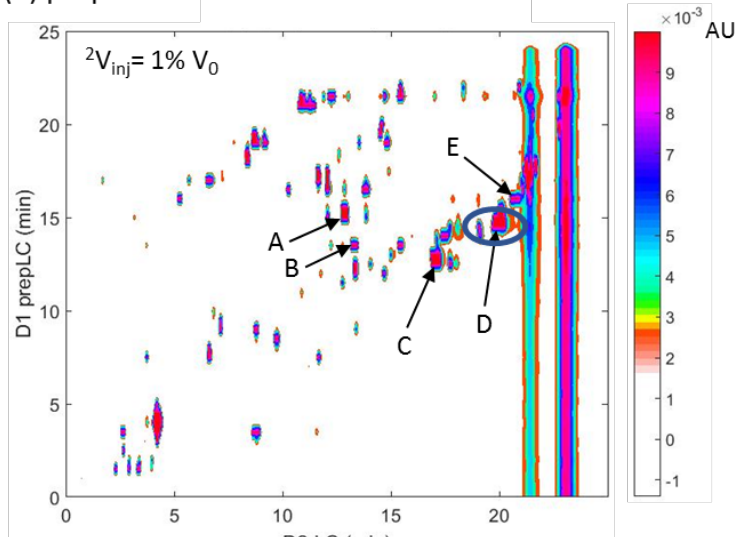

(c) prepLCxLC

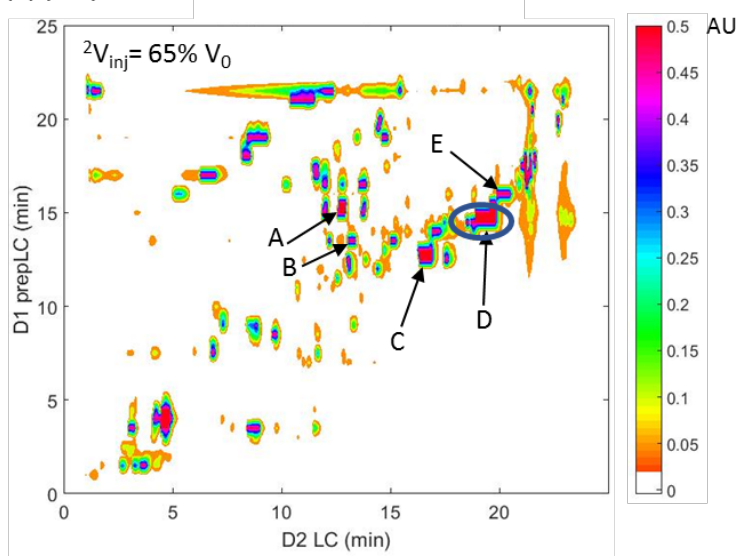

(b) CPCxLC

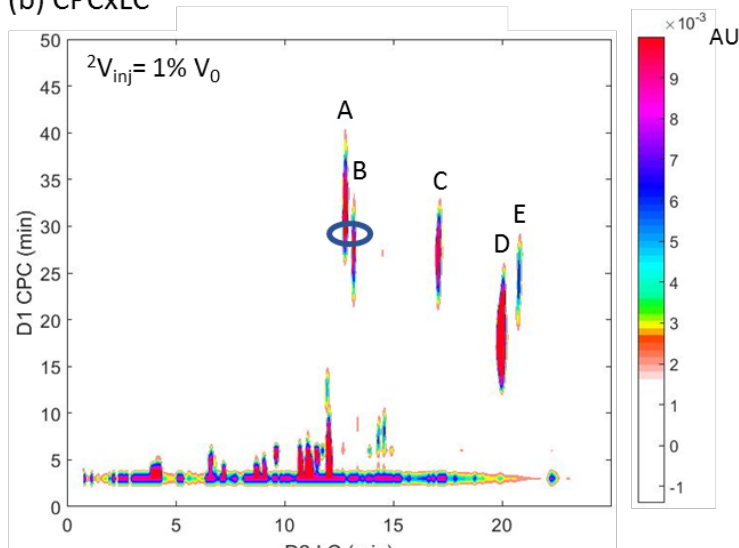

(d) $\mathrm{CPCxLC}$

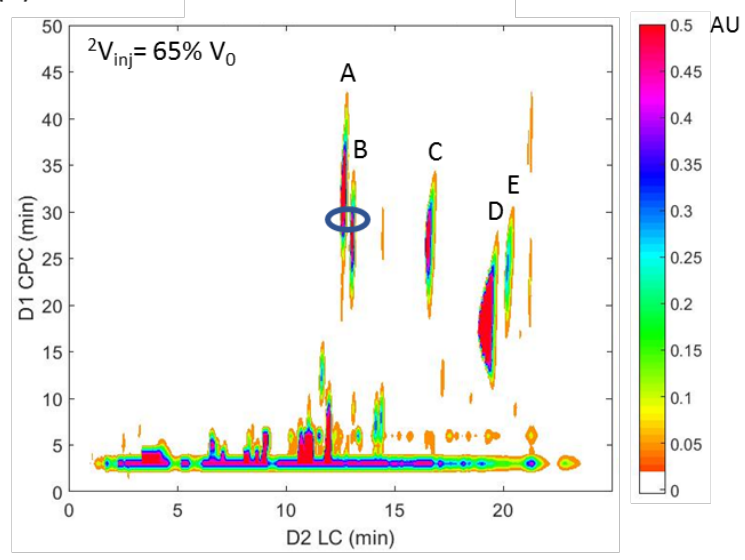

Figure 3. 2D-contour plots of off-line comprehensive two-dimensional separations (a) prepLCXLC at

519 analytical step (b) CPCxLC at analytical step (c) prepLCxLC at loading step (d) CPCxLC at loading step. In circles are the critical pairs of each configuration. 
(a) prepLCxLC fraction \#30

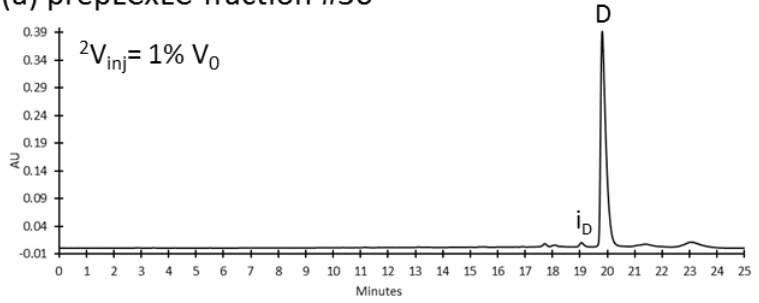

(c) prepLCxLC fraction \#30

521

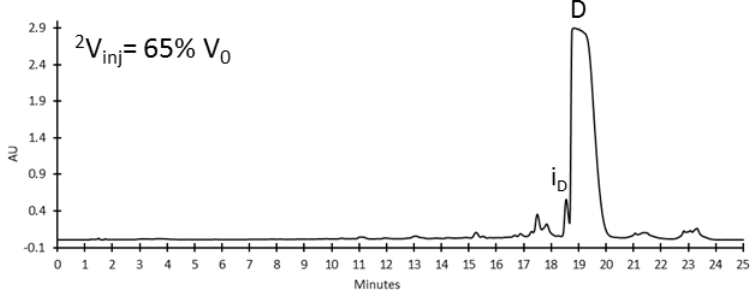

(b) CPCXLC fraction \#30

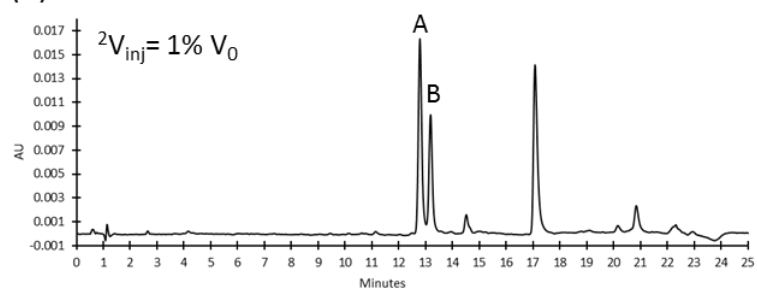

(d) CPCxLC fraction \#30

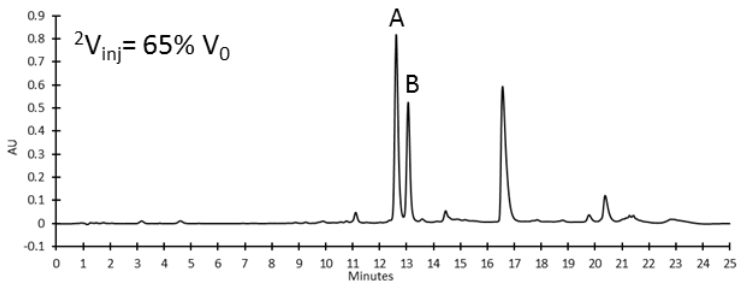

522 Figure 4. 2nd-dimension LC chromatograms at analytical step of (a) fraction \#30 of prepLC (b) fraction

523 \#30 of CPC and 2nd-dimension LC chromatograms at loading step of (c) fraction \#30 of prepLC (d)

524 fraction \#30 of CPC. 
525 Figure 1. HPLC analysis of the crude sample on Phenyl-Hexyl column (150 mm x $4.6 \mathrm{~mm}$ i.d., $5 \mu \mathrm{m}$ ),

526 mobile phase of water $+0.1 \%$ of formic acid $(A)$ and acetonitrile $+0.1 \%$ of formic acid (B) in gradient

527 mode: $12 \%$ B for $1.1 \mathrm{~min}, 12$ to $35 \% \mathrm{~B}$ in $18.4 \mathrm{~min}, 35$ to $95 \%$ B for $0.8 \mathrm{~min}$, flow-rate $2 \mathrm{ml} / \mathrm{min}$,

528 detection $330 \mathrm{~nm}$.

529

530 Figure 2. Two-dimensional process with CPC and prepLC as first dimensions and LC as second

531 dimension with the various sizes corresponding to the successive development steps.

532

533 Figure 3. 2D-contour plots of off-line comprehensive two-dimensional separations (a) prepLCxLC at

534 analytical step (b) CPCxLC at analytical step (c) prepLCxLC at loading step (d) CPCxLC at loading step.

535 In circles are the critical pairs of each configuration.

536

537 Figure 4. 2nd-dimension LC chromatograms at analytical step of (a) fraction \#30 of prepLC (b) fraction

$538 \# 30$ of CPC and 2nd-dimension LC chromatograms at loading step of (c) fraction \#30 of prepLC (d)

539 fraction \#30 of CPC. 


\begin{tabular}{|c|c|c|}
\hline & prepLCxLC & CPCxLC \\
\hline Strategy & $\begin{array}{l}\text { Spread the solutes over a large } \\
\text { separation space to get better } \\
\text { chance to separate targets }\end{array}$ & $\begin{array}{c}\text { Separate target compounds from } \\
\text { the others by finely tuning the } \\
\text { solvent system }\end{array}$ \\
\hline Development method & Fast & Slow \\
\hline${ }^{1} \mathrm{D}$ column & $150 \mathrm{~mm} \times 10 \mathrm{~mm}$ & $35.8 \mathrm{ml}$ rotor \\
\hline $\begin{array}{l}\text { Cost and duration of first } \\
\text { dimension (including } \\
\text { equilibration, blanks) }\end{array}$ & $\begin{array}{c}475 \mathrm{ml} \text { of solvent } \\
50 \mathrm{~min}\end{array}$ & $\begin{array}{l}325 \mathrm{ml} \text { of solvent } \\
65 \mathrm{~min}\end{array}$ \\
\hline $\mathrm{n}_{\mathrm{c}, 2 \mathrm{D}, \text { theo }}$ & 900 & $<300$ \\
\hline Selectivity & Low & High \\
\hline \multirow[t]{2}{*}{${ }^{2} \mathrm{D}$ column load } & $19 \%$ of column dead volume & $313 \%$ of column dead volume \\
\hline & \multicolumn{2}{|c|}{ using a $150 \mathrm{~mm} \times 10 \mathrm{~mm}^{2} \mathrm{D}$ column } \\
\hline Fractions volume & $1.5 \mathrm{ml}$ & $24 \mathrm{ml}$ \\
\hline${ }^{1} \mathrm{D}$ sampling time & $0.16 \mathrm{~min}$ & $4.8 \mathrm{~min}$ \\
\hline Comprehensive strategy & 157 fractions & 11 fractions \\
\hline \multirow[t]{2}{*}{$\begin{array}{c}\text { Selective comprehensive } \\
\text { strategy ( } 5 \text { targets) }\end{array}$} & 29 fractions & 7 fractions \\
\hline & \multicolumn{2}{|c|}{ using alternative ${ }^{2} D$ column size } \\
\hline Second dimension alternative & Column $150 \mathrm{~mm}$ x $19 \mathrm{~mm}$ & Column $150 \mathrm{~mm}$ x $4.6 \mathrm{~mm}$ \\
\hline Fractions volume & $4.75 \mathrm{ml}$ & $5 \mathrm{ml}$ \\
\hline Comprehensive strategy & 50 fractions & 50 fractions \\
\hline $\begin{array}{l}\text { Selective comprehensive } \\
\text { strategy ( } 5 \text { targets) }\end{array}$ & 9 fractions & 34 fractions \\
\hline
\end{tabular}

542 